\title{
P 084 HEALTH PROFESSIONALS' PERSPECTIVES ABOUT ADVANCE CARE PLANNING IN PARKINSON'S DISEASE: A QUALITATIVE STUDY
}

Timothy Jackson, ${ }^{1}$ Alexander Gerhard, ${ }^{2}$ Catherine Walshe ${ }^{3} .{ }^{1}$ Department of Palliative Care, Salford Royal NHS Foundation Trust, Salford, UK; ${ }^{2}$ Institute of Brain, Behaviour and Mental Health, The University of Manchester, UK; ${ }^{3}$ International Observatory on End of Life Care, Division of Health Research, Lancaster University, UK

\subsection{6/bmjspcare-2014-000654.125}

Background People with Parkinson's disease face repeated hospital admissions and medical interventions in the last year of life. Advance care planning (ACP) is advocated, aiming to empower patients by engaging them in decisions about their future. There is little evidence about the optimum timing and content of ACP or how this process should be coordinated for those with Parkinson's disease.

Aim To discover the views of health professionals on best practice for ACP in Parkinson's disease and how organisations might better support multi-disciplinary teams to undertake ACP.

Method Qualitative design, using semi-structured interviews with a purposive sample of health professionals, with experience of Parkinson's disease or ACP in other neurological conditions $(n=16)$. Participants were recruited through neurology, elderly medicine, and palliative care departments at three organisations (a tertiary neurology centre, teaching hospital and a hospice). Interviews were audio-recorded and transcribed. Iterative thematic analysis comprised familiarisation, coding and categorisation. The study received all necessary governance approvals.

Results There was consensus that prognostic indicators and patient cues should prompt discussions at an appropriate time for each individual. Potential discussion areas included preferred place of care, artificial nutrition and hydration, and future hospitalisation. Participants felt the organisational approach to ACP should be better coordinated through improved collaborative working across different settings and use of tools such as electronic palliative care records. Some felt professionals should be offered further training, but most felt that different aspects of ACP could be undertaken by a range of medical, nursing and other staff.

Conclusion ACP should be a personalised, flexible and dynamic process for patients with Parkinson's disease. Organisations should support different staff groups to undertake ACP, providing appropriate tools, training and resources. Better joint working between palliative care, neurology and elderly care teams and allied health professionals should be encouraged and supported. 